\title{
JOSEPH FELS RITT
}

All Columbia College mathematics students came under the influence and scrutiny of J. F. Ritt in his introductory course on the solution of differential equations. To many teachers this subject presents no particular fascination; we appear to have here a not too well connected collection of facts and methods whose presentation would not at first glance seem very enlivening. But Ritt gave this material a characteristic glow. In all branches of mathematics he was an avid student of the past; in the field of differential equations he was a particularly distinguished authority. In his classes his own mind recreated the greatness of a golden period of our subject with special emphasis on the contributions of the French school, ever dear to him. It was in this course that the writer first met Professor Ritt. In the ensuing twenty-four years, my relations with him were constant as a younger colleague and friend. His recent death has terminated a career of outstanding distinction. His scientific achievements in the contemporary mathematical scene are truly impressive. A necessarily brief summary of his life work is given in the later pages of this article. It should be recorded here that on numerous occasions Ritt expressed the belief that a man's work should speak for him and that vitae or obituaries were artificial and superfluous commentaries on the ebb and flow of history. ${ }^{1}$

Ritt was born on August 23, 1893 in New York City. His early education in the city included two years at the College of the City of New York. He obtained the degree of Bachelor of Arts from George Washington University in 1913. The same institution honored him with the bestowal of the degree of Doctor of Science in 1932. After some years as a graduate student at Columbia University, where he came under the influence of Professors Fite and Kasner, he obtained in 1917 the Ph.D. for a work which is fresh even now. Ritt referred frequently and with affectionate pride to his dissertation. Except for two years spent as a Master Computer in the Ordnance Department, (1918-1919), he spent the remainder of his life at Columbia. In 1928 he married Miss Estelle Fine who for over twenty years was her husband's devoted companion. Together they labored at the preparation of his many manuscripts, they relaxed in travel to various

\footnotetext{
${ }^{1}$ In the preparation of the manuscript valuable help was received from many friends. Mrs. Ritt very kindly checked the bibliography. The members of the department of mathematics at Columbia as well as E. L. Post and B. P. Gill made valuable suggestions. Two paragraphs on some deeper aspects of the work in differential equations (low-power theorem, difference equations) are due to Ellis Kolchin.
} 
countries and around the world, and they shared every success which came to him. The end came suddenly and without warning to his friends, on January 5,1951 , at a time when he was engaged in some of the most important work of his life.

Ritt had an enviable reputation as a teacher. His lectures were etched on the minds of his hearers who carried away a feeling of masterfulness. His wit was out of the ordinary. Both in a completely impromptu sally and in studied and carefully polished excursions, the unexpectedness and sure impact of his phrases were unmatched. His colleagues referred to them as ritticisms. In the writing of his papers he was capable of devoting weeks of worry to the proper balance of a single thought occurring in one paragraph. This will reveal the dogged tenacity of his mind-at once a strong quality in his own work, and yet not without elements of weakness. His memory was astonishing. Being much taken with the tempo of Mr. Churchill's prose, he memorized in a single reading whole sections of speeches made by the latter during the dark days of the war, and would regale his hearers months and years later with their recital. Although thus endowed, he was definitely not encyclopedic in mathematics. His wise comment on this was: "A man will know as much as he needs." He was an amateur of languages although he did not derive social enjoyment from prolonged conversation in other idioms. For example, he learned to read mathematical Russian before that became fashionable.

He laid the greatest emphasis on the question of style in mathematical papers. His ideas had been developed from intensive study of the writings of the masters of the eighteenth and nineteenth centuries. Here he found a distinction which one does not readily discover in the desiccated writing of our own day,- carried on in the shadow of a hawk-like editor who parsimoniously counts the pages to his petitioner. All of Ritt's books and papers were written with the greatest stylistic care. He did not consider his contributions as being local in time but rather definitive; for that reason he wished them to be in final form. At times the striving for smoothness of pace and ease of ascent may impress the reader with technical craftsmanship rather than well-balanced and intuitive artistry. The fact is that both are present in all of his writing.

The excellence of his work was recognized early, by his University, by the professional society of which he was a member, and by the world at large. At the age of 39 he was Colloquium Lecturer of the Society. Shortly after, he was elected to membership in the $\mathrm{Na}$ tional Academy of Sciences. Two of his books appear in the Colloquium Publications, an unusual honor. He was vice-president of the 
Society from 1938 to 1940 . Other elected positions he held in the Society were: Trustee, 1923-1924; member of the Council, 19261928; representative on the Board of Editors of the American Journal of Mathematics, 1936-1940; and member of the Colloquium Editorial Committee, 1943-1948. His works received acclaim in Europe. For example, Bieberbach in his invited address to the 1932 International Congress in Zürich devoted one-half of his lecture to a discussion of his writings. At home he had a score of young students of distinction. During the last decade he occupied the Davies Chair of Mathematics. At the 1950 International Congress in Cambridge, he gave an invited address on his latest work in which he developed a Lie theory of differential groups. Only one mark of esteem escaped him. He was never the recipient of one of our great prizes. There can be no doubt of the fact that this was a source of more than disappointment to him. As one views his writings both as a young man and later as a mature scientist, one is compelled to admit with generosity his claim to fame. That he failed on successive occasions to receive this particular symbol of greatness suggests a weakness in our social organization. It is true that our field of endeavor is a blessed paradise compared to certain others in this matter. But perhaps our situation is not ideal. Is it not possible that we have too few prizes? or perhaps too many?

From acquaintance with the grown man, one would surmise that the boy was shy, probably excessively so. As his life was governed by a strict and occasionally inflexible code, his sensitive spirit was never reconciled to the chaotic organism which is society. During the early years, the growing boy became conscious of his own genius and with this developed a tremendously strong feeling of independence. The need for collaboration and association with a scientific school of his day was not present since all alone he could evaluate, plan, and execute. In order to satiate the unquenchable universal need for companionship, he turned to the great of all time. Here one could find the noblest inspiration as well as freedom from strife and uncertainty. The fascination of the grandeur of bygone days had an immeasurable influence on him. His heroes were Lagrange, Laplace, Abel, Jacobi, Liouville, Cauchy, Poincaré, and Hilbert. From them he derived standards of excellence; of them he hoped to be worthy. His study of mathematics was through the man. This is the normal approach in the arts and letters but is much less usual in the sciences. Most of us appreciate the man through a preliminary mastery of the subject.

His own inclinations and abilities, and the moulding forces in his 
personality which have been adumbrated above, channelled his creative energies into the field of classicism. His media are complex function theory of the nineteenth century and differential equations. Much of his work could have been written a half century earlier. Instinctively he sought for problems to solve. His papers and numerous conversations underline this constant search for problems of importance in a completely established domain beyond the shadow of controversy. The "modern" exploratory spirit in algebra, topology, or linear spaces was not for him. He did not understand it nor did he wish to. The Lebesgue integral he begrudgingly recognized. But fields of characteristic $p$ were special objects of scorn. That all of classic number theory was bound up with them he preferred not to contemplate. Yet his own instincts were so sure that only once did this disregard for new developments conduce to an imperfection in his written work. In his chosen specialties he exhibited unmatched virtuosity. The problems were so natural that one marvelled that they had not received attention before. The solution was carried through like the traditionally perfect surgical operation. Yet with this distrust of the modern, and with this emphasis on classical analysis, it is nevertheless a fact which is borne out by his lifelong work that Ritt was fascinated by the elemental idea from which springs all of contemporary algebra-the notion of structure. Some of his very best papers lead to a unique factorization theorem, the very essence of rich algebra. Details will make this clear shortly. A favorite operation which comes under his scrutiny is that of composition of functions (forming $f(g(z))$ from $f(z)$ and $g(z)$ ) which gives an associative "multiplication." Finally, in algebraic differential equations the theory of the manifold of solutions is described in terms of intersection of prime ideals.

The dissertation [5 $]^{2}$ was presented to the Society in April 1916. Here are considered the properties of the linear homogeneous differential operator of infinite order with constant coefficients: $A$ $=\prod_{1}^{\infty}\left(1-D / a_{i}\right)$ where $\sum_{1}^{\infty}\left|a_{i}\right|^{-1}<\infty$ and $D$ represents $d / d z$. Application of $A$ to an analytic function $f(z)$ yields again such a function. Any solution of $A \Phi(z)=0$ which is analytic on the circumference of a circle is analytic throughout the interior of the circle, hence no solution may have an isolated singularity. These two results will give an idea of the material developed.

After a brief period of orientation come two brilliant papers Prime and composite polynomials [14] and On algebraic functions which can be expressed in terms of radicals [15]. In the first, a poly-

\footnotetext{
${ }^{2}$ Numbers in brackets refer to the bibliography at the end of the paper.
} 
nomial $F(z)$ is called composite if there exist polynomials $\phi_{1}(z)$ and $\phi_{2}(z)$ of degree higher than one such that $F(z)=\phi_{1}\left(\phi_{2}(z)\right)$. Thus we are considering the problem of factoring polynomials into primes$F=\phi_{1} \phi_{2} \cdots \phi_{8}$. It is proved that any two decompositions contain the same number of polynomials and that the degrees are the same in both decompositions. Essentially distinct decompositions are completely analyzed. In the second, Ritt determines all algebraic functions $w$ defined by a polynomial $F(w, z)=0$ which can be expressed in terms of $z$ by means of radicals. Here is certainly a beautiful problem in the most classic tradition! As the author put it: "Why the results obtained here should not have been found before this time is a question which has puzzled us as much as it will puzzle the reader." For the case of genus zero, the rational functions whose inverse can be expressed in terms of radicals can be obtained from the functions $z^{n} ; f_{n}(z)$ where $\cos n u=f_{n}(\cos u)$; the rational functions which occur in the formulas for the transformations of the periods of the function $\wp(u)$; and certain other functions associated with elliptic functions in the lemniscatic $\left(g_{3}=0\right)$ and the equianharmonic $\left(g_{2}=0\right)$ cases. In both of these papers we have masterful treatment of monodromy groups of Riemann surfaces, in particular of those associated with trigonometric polynomials and elliptic functions. The same techniques are used in one of Ritt's most important papers Permutable rational functions [18]. The problem is to find conditions under which rational functions $\phi(z)$ and $\psi(z)$ satisfy $\phi(\psi(z))=\psi(\phi(z))$. This paper finds Ritt competing with some of his contemporaries in the solution of a specific problem for probably the only time in his scientific life. Julia and Fatou in France had obtained preliminary results in the direction of a solution, but Ritt's results, arrived at by completely different methods, are much more definitive. This paper marks the high point of the first period in his career. The particular problem with which it deals was probably more in the public eye than those which follow it. After this publication it was clearly evident that a master had stepped forth.

We turn now to his contributions in the theory of elementary functions. The field was essentially in the state in which Liouville had left it eighty-five years before. Ritt's great tour de force here was the determination of the structure of the elementary functions $y=f(x)$ whose inverses $x=g(y)$ are also elementary. He showed in a tough paper [22] that they are precisely the functions $f(x)$ for which a "factorization" exists: $f=\phi_{1} \phi_{2} \cdots \phi_{s}$, where the functions $\phi_{i}(x)$ are either $e(x), \log x$, or an algebraic function of $x$. Any such function $f(x)$ evidently has an elementary inverse; note for example that the 
function $x+\log x$ does not have an elementary inverse. He showed as well that the elliptic integrals $w=g(z)$ do not satisfy an elementary equation in two variables, $F(w, z)=0$, thus improving on a result of Liouville who had demonstrated that the integrals themselves were not elementary, and proving that the elliptic functions are not elementary. We note here once more the fascination with the notion of factorization. The methods of attack based on a classification of the elementary functions are due to Liouville. One can trace the influence of this classification several years later in the totally unconnected field of algebraic differential equations. In the latter, the coming to grips with the problem is based on a very ingenious classification of rank of differential polynomial. Ritt offered a course of lectures on elementary functions at frequent intervals. In 1947 he wrote a short monograph on the subject [C] making available for the first time in book form the salient points of this theory. It is strange to think of writing a book on a branch of mathematics which almost certainly will be the definitive work for the next fifty to seventy-five years. Yet this seems to be the case here. It is probable, nevertheless, that Ritt overemphasized the importance of this subject. It is true, as he contended, that younger students deserve to have the answer to various questions which arise in the calculus; but the elaboration of this answer contributes little to the opening of vistas into the domain of vibrantly living mathematics.

In the next few years most of the papers deal with exponential polynomials. The problem of factoring (once more!) the expression $\sum_{1}^{n} a_{i} e\left(\alpha_{i} z\right)$ into irreducible exponential polynomials is completely solved [31]. In another paper it is shown that if every zero of one exponential polynomial is a zero of a second polynomial, the quotient of the second by the first is an exponential polynomial [35]. In a third paper of this series it is established that if $w$ is a uniform function satisfying $\sum_{0}^{n} P_{i} w^{i}=0$ where the $P_{i}$ are exponential polynomials, then $w$ is the quotient of two exponential polynomials [34].

We come now to the outstanding work in Ritt's career, to which he devoted almost twenty years of his life and for which above all else he will be remembered decades hence-his creation of a theory of ordinary and partial algebraic differential equations. The basic theory appears full-fledged in his paper Manifolds of functions defined by systems of algebraic differential equations [37]. In the score of years that follow, there is a flow of some twenty papers on the subject, from his own pen as well as a large number from his students. Every aspect of the theory is studied in detail. It is impossible for several reasons to give here an extensive review of the material covered. The 
most important portions of it will be found in his two Colloquium Publications, volumes 14 and 33 [A, D]. For a preliminary orientation, one might recommend chapter I of volume 33 as well as an address delivered at the semi-centennial celebration of the Society, Algebraic aspects of the theory of differential equations [49]. We shall make a few remarks concerning the situation under scrutiny. Ritt considers differential polynomials or forms in the unknown functions $y_{1}, \cdots, y_{n}$ and their derivatives with coefficients which are functions meromorphic in some domain. Given a system $\Sigma$ of such forms, he shows that there exists a finite subsystem of $\Sigma$ whose manifold of solutions is identical with that of $\Sigma$. Furthermore, if a form $G$ vanishes for every solution of the system of forms $H_{1}, \cdots, H_{r}$, then some power of $G$ is a linear combination of the $H_{i}$ and their derivatives. Define a basis of $\Sigma$ to be a finite subset $\Phi$ of $\Sigma$ such that every $G$ in $\Sigma$ has the property that for some positive integer $p, G^{p}$ is a linear combination of the forms in $\Phi$ and their derivatives. It then follows from the above that every infinite system of forms has a basis.

Next, Ritt considers the question of reducibility. A system $\Sigma$ is said to be reducible if there exist two forms $G$ and $H$ such that every solution of $\Sigma$ is a solution of $G H$ while some solutions of $\Sigma$ are not solutions of $G$, and others are not solutions of $H$. There follows then the fundamental theorem that every system $\Sigma$ is equivalent to a finite set of irreducible systems. Let $\{\Sigma\}$ represent the perfect differential ideal generated by $\Sigma$. Let $\mathfrak{M}$ represent the manifold of solutions of $\Sigma$ and hence of $\{\Sigma\}$. Suppose the essential irreducible systems to which $\Sigma$ is equivalent are $\Sigma_{1}, \cdots, \Sigma_{p}$; suppose $\mathfrak{M}_{i}$ is the manifold of $\Sigma_{i}$. Then clearly $\left\{\Sigma_{i}\right\}$ is a prime ideal. Also, we have $\mathfrak{M}=\mathfrak{M}_{1} \cup \ldots \cup \mathfrak{M}_{p}$ by the above fundamental theorem. But this statement is precisely equivalent to the statement $\{\Sigma\}=\left\{\Sigma_{1}\right\} \cap \ldots$ $\cap\left\{\Sigma_{p}\right\}$. This is the theorem on the unique factorization into primes of a given perfect ideal. The analogy to systems of polynomials in the theory of ordinary polynomials will now be clearer. One should keep in mind however that the introduction of differentiation introduces entirely new phenomena and thus the present theory is not a "mere" generalization of polynomial ideal theory.

Ritt defines next the notions of a general solution of a single equation $A=0$. One may advance the thesis that all his work on differential equations owes its origin to the very unsatisfactory state of the nature of general and singular solutions in the literature at large and more specifically in the writings of Laplace, Lagrange, and Poisson which were most carefully examined by Ritt. Let $A$ be algebraically irreducible and suppose $A$ involves some particular $y_{j}$. 
Let $S_{j}$ be the partial derivative of $A$ with respect to the highest derivative of $y_{j}$ occurring in $A$. Let $\{A\}=\left\{\Sigma_{1}\right\} \cap \cdots \cap\left\{\Sigma_{p}\right\}$ be the factorization of $\{A\}$ into prime ideals. Then there is precisely one $\left\{\Sigma_{i}\right\}$ whose solutions do not cause all $S_{j}$ to vanish. Furthermore this $\left\{\Sigma_{i}\right\}$ is independent of $j$. The manifold of $\left\{\Sigma_{i}\right\}$ is called the general solution of $A=0$. The remaining manifolds determined by the $\left\{\Sigma_{k}\right\}, k \neq i$, constitute the singular manifolds.

Thus all the pivotal results were obtained by him in the early stages of his program. However, the definitive ideal-theoretic formulation of the theory (intersection theorem) is due to Raudenbush, one of his students, who discovered it shortly after completing his dissertation under him. It is a tribute to Ritt's perfectionism that he considered that he had failed in not perceiving himself the primary role played by ideal theory in his problem. Yet failure it most certainly was not. Rather is was a poignant experience that can arise only in a genuinely rich scientific career.

A second complex of results in his theory was secured by him in papers dating from 1936 to 1945 . He proved that every irreducible component of the manifold of a differential polynomial $A$ is the general solution of some irreducible differential polynomial $B$. Furthermore he gave a simple algorithm (the "low power theorem") by which one can decide, given two differential polynomials $A$ and $B$, when the general solution of $B$ is a component of the manifold of $A$. Also, he attempted to discover an algorithm for deciding when a given solution of $A$ belongs to the general solution of $A$; this is evidently a very difficult question. For the case in which $A$ is an ordinary differential polynomial in one unknown and is of order $\leqq 2$, he gave a complete answer, albeit not a simple one. These and certain other results were all established by the construction of certain formal power series solutions (the "polygon process"), and seem to lie deeper than the earlier part of the theory.

Shortly after he launched his theory of algebraic differential equations he perceived the possibility of an analogous theory for algebraic difference equations. With the occasional collaboration of some of his students he succeeded in establishing such a theory. Actually this theory deals with equations more general than difference equations. For example it handles substitutional equations $P(x, y(x), y(\phi(x)), \cdots, y(\phi(\cdots \phi(x) \cdots)))=0$, where $P$ is a polynomial in $y(x), y(\phi(x)), \cdots, y(\phi(\cdots \phi(x) \cdots))$, and $\phi(x)$ is a given function, or more generally equations $P\left(y, \sigma y, \cdots, \sigma^{n} y\right)=0$, where $P$ is a polynomial with coefficients in a field $K$ and $\sigma$ is an endomorphism of $K$. 
The last phase of his writing began three years ago. Ritt came upon problems which generalize the Lie theory. Consider an infinite series $P_{1}+P_{2}+\cdots$ where the $P_{i}$ are homogeneous differential polynomials of degree $i$ in the indeterminates $y_{1}, \ldots, y_{p}$ and their derivatives with coefficients in a differential field. (Derivatives are written $y_{i j}$, or in case $p=1$ as $y_{j}$.) One may make a substitution by replacing each $y_{i}$ by an expression of the above type in the new indeterminates $y_{i}^{\prime}, \cdots, y_{p}^{\prime}$. Specifically, consider two sets of indeterminates $u_{i}, v_{i}$, $i=1, \cdots, n$, and $n$ series of this type $A_{i}\left(u_{i}, \cdots, u_{n} ; v_{i}, \cdots, v_{n}\right)$, $i=1, \cdots, n$. Write the above for short as $A(u, v)$. The equation $A[A(u, v), w]=A[u, A(v, w)]$ states that $A$ is an associative operation. Consider as an example $A(u, v)=u+v$. A differential group is a set of power series $z_{i}=A_{i}(u, v)$ such that each $A_{i}$ starts with the polynomial $u_{i}+v_{i} ; z_{i}=u_{i}$ if $v=0$ and $z_{i}=v_{i}$ if $u=0$; the $A_{i}(u, v)$ have the associative property described above.

In his first paper [63], Ritt determines all groups of order 1, that is, those for which $n=1$. They are the additive group $z=u+v$ and the group of substitutions. The latter may be made more explicit as follows: If $z(x)=u(x)+v[x+u(x)]$, then the usual Taylor series gives $z=u+v+\sum_{n=1}^{\infty} v_{n} u^{n} / n !$; if one sets $z^{\prime}(x)=x+z(x), u^{\prime}(x)=x$ $+u(x), v^{\prime}(x)=x+v(x)$, then $z^{\prime}(x)=v^{\prime}\left[u^{\prime}(x)\right]$. Thus at this point we already see a difference between these results and those of the classical Lie theory. The case of groups of order 2 is even more revealing. In his invited address to the 1950 International Congress in Cambridge, Ritt described thirteen distinct types of groups of this order. In another paper [64], he gives identities satisfied by the structure constants of differential groups which resemble those given in Lie's third fundamental theorem. Other results not known to the writer are still on the printer's desk, awaiting publication at this very moment. The theory had just entered upon maturity when its creator laid down his pen. This and the older work is our inheritance.

\section{BiBLIOGRAPHY BOOKS}

A. Differential equations from the algebraic standpoint, Amer. Math. Soc. Colloquium Publications, vol. 14, 1932, 173 pp.

B. Theory of functions, King's Crown Press, 1947, $181 \mathrm{pp}$.

C. Integration in finite terms, Columbia University Press, 1948, $100 \mathrm{pp}$.

D. Differential algebra, Amer. Math. Soc. Colloquium Publications, vol. 33, 1950, 186 pp.

\section{PAPERS}

1. A control for least square solutions, Astronomical Journal vol. 28 (1913) pp. 7374. 
2. Note sur la fonction $\sin [(n+1)$ arc $\cos x]$, Nouvelles Annales de Mathématiques (4) vol. 14 (1914) pp. 184-186.

3. On certain real solutions of Babbage's functional equation, Ann. of Math. vol. 17 (1916) pp. 113-122. 23.

4. On the derivatives of a function at a point, Ann. of Math. vol. 18 (1916) pp. 18-

5. On a general class of linear homogeneous differential equations of infinite order with constant coefficients. Preceded by a note on The resolution into partial fractions of the reciprocal of an entire function of genus zero, Dissertation, Trans. Amer. Math. Soc. vol. 18 (1917) pp. 21-49.

6. On the differentiability of asymptotic series, Bull. Amer. Math. Soc. vol. 24 (1918) pp. 225-227.

7. Sur l'itération des fonctions rationelles, C. R. Acad. Sci. Paris vol. 166 (1918) pp. $380-381$.

8. On the differentiability of the solution of a differential equation with respect to a parameter, Ann. of Math. vol. 20 (1919) pp. 289-291.

9. On the iteration of rational functions, Trans. Amer. Math. Soc. vol. 21 (1920) pp. 313-320.

10. On weighting factor curves in flat fire, Journal of United States Artillery vol. 53 (1920) pp. 404-410.

11. On the conformal mapping of a region into a part of itself, Ann. of Math. vol. 22 (1921) pp. 157-160.

12. Note on equal continuity, Bull. Amer. Math. Soc. vol. 27 (1921) pp. 351-353.

13. Periodic functions with a multiplication theorem, Trans. Amer. Math. Soc. vol. 23 (1922) pp. 16-25.

14. Prime and composite polynomials, Trans. Amer. Math. Soc. vol. 23 (1922) pp. 51-66.

15. On algebraic functions which can be expressed in terms of radicals, Trans. Amer. Math. Soc. vol. 24 (1922) pp. 21-30.

16. Sur les fonctions rationnelles permutables, C. R. Acad. Sci. Paris vol. 176 (1923) pp. $60-68$.

17. On the integrals of elementary functions, Trans. Amer. Math. Soc. vol. 25 (1923) pp. 211-222.

18. Permutable rational functions, Trans. Amer. Math. Soc. vol. 25 (1923) pp. 399-448.

19. Equivalent rational substitutions, Trans. Amer. Math. Soc. vol. 26 (1924) pp. 221-229.

20. Analytic functions and periodicity, Bull. Amer. Math. Soc. vol. 30 (1924) pp. 406-409.

21. Note on the Dirichlet series with complex exponents, Ann. of Math. vol. 26 (1924) p. 144.

22. Elementary functions and their inverses, Trans. Amer. Math. Soc. vol. 27 (1925) pp. 68-90.

23. New proofs of the two well-known theorems on quadratic forms, Ann. of Math. vol. 26 (1925) pp. 202-204.

24. Sur les fonctions méromorphes qui admettent un theorème d'addition ou de multiplication, C. R. Acad. Sci. Paris vol. 182 (1926) pp. 201-202.

25. Simplification de la méthode de Liouville dans la théorie des fonctions élémentaires, C. R. Acad. Sci. Paris, vol. 183 (1926) pp. 331-332.

26. Transcendental transcendency of certain functions of Poincarê, Math. Ann. vol. 95 (1926) pp. 671-682. 
27. On the integration in finite terms of linear differential equations of the second order, Bull. Amer. Math. Soc. vol. 33 (1927) pp. 51-57.

28. (With Eli Gourin) An assemblage-theoretic proof of the existence of transcendentally transcendental functions, Bull. Amer. Math. Soc. vol. 33 (1927) pp. 182-184.

29. Meromorphic functions with addition or multiplication theorems, Trans. Amer. Math. Soc. vol. 29 (1927) pp. 341-360.

30. Real functions with algebraic addition theorems, Trans. Amer. Math. Soc. vol. 29 (1927) pp. 361-368.

31. A factorization theory for functions $\sum_{i=1}^{n} a_{i} e^{a_{i}^{x}}$, Trans. Amer. Math. Soc. vol. 29 (1927) pp. 584-596.

32. On certain points in the theory of Dirichlet series, Amer. J. Math. vol. 50 (1928) pp. 73-86.

33. Calculus, differential and integral, Encyclopedia Britannica 14th ed., vol. 4, 1929, pp. 555-558.

34. Algebraic combinations of exponentials, Trans. Amer. Math. Soc. vol. 31 (1929) pp. 654-679.

35. On the zeros of exponential polynomials, Trans. Amer. Math. Soc. vol. 31 (1929) pp. 680-686.

36. On a certain ring of functions of two variables, Trans. Amer. Math. Soc. vol. 32 (1930) pp. 155-170.

37. Manifolds of functions defined by systems of algebraic differential equations, Trans. Amer. Math. Soc. vol. 32 (1930) pp. 569-598.

38. Representation of analytic functions as infinite products, Math. Zeit. vol. 32 (1930) pp. 1-3.

39. Systems of algebraic differential equations, Proc. Nat. Acad. Sci. U.S.A. vol. 17 (1931) pp. 366-368.

40. Integral functions obtained by compounding polynomials, Bull. Amer. Math. Soc. vol. 39 (1933) pp. 627-632.

41. (With J. L. Doob) Systems of algebraic difference equations, Amer. J. Math. vol. 55 (1933) pp. 505-514. 308.

42. Algebraic difference equations, Bull. Amer. Math. Soc. vol. 40 (1934) pp. 303-

43. Systems of algebraic differential equations, Ann. of Math. (2) vol. 36 (1935) pp. 293-302.

44. Jacobi's problem on the order of a system of differential equations, Ann. of Math. (2) vol. 36 (1935) pp. 303-312.

45. Indeterminate expressions involving a function and its derivatives, Monatshefte für Mathematik und Physik vol. 43 (1936) pp. 97-104.

46. On the singular solutions of algebraic differential equations, Ann. of Math. vol. 37 (1936) pp. 552-617.

47. On certain points in the theory of algebraic differential equations, Amer. J. Math. vol. 60 (1938) pp. 1-43.

48. Systems of differential equations I. Theory of ideals, Amer. J. Math. vol. 60 (1938) pp. 535-548.

49. Algebraic aspects of the theory of differential equations, Amer. Math. Soc. Semicentennial Publications, vol. 2, 1938, pp. 35-55.

50. On ideals of differential polynomials, Proc. Nat. Acad. Sci. U.S.A. vol. 25 (1939) pp. 90-91.

51. On the intersections of algebraic differential manifolds, Proc. Nat. Acad. Sci. U.S.A. vol. 25 (1939) pp. 214-215. 
52. (With H. W. Raudenbush, Jr.) Ideal theory and algebraic difference equations, Trans. Amer. Math. Soc. vol. 46 (1939) pp. 445-452.

53. (With E. R. Kolchin) On certain ideals of differential polynomials, Bull. Amer. Math. Soc. vol. 45 (1939) pp. 895-898.

54. On the intersections of irreducible components in the manifold of a differential polynomial, Proc. Nat. Acad. Sci. U.S.A. vol. 26 (1940) pp. 354-356.

55. On a type of algebraic differential manifold, Trans. Amer. Math. Soc. vol. 48 (1940) pp. 542-552.

56. Complete difference ideals, Amer. J. Math. vol. 63 (1941) pp. 681-690.

57. Bezout's theorem and algebraic differential equations, Trans. Amer. Math. Soc. vol. 53 (1943) pp. 74-82.

58. A family of functions and its theory of contact, Bull. Amer. Math. Soc. vol. 49 (1943) pp. 109-113.

59. On the manifolds of partial differential polynomials, Ann. of Math. vol. 46 (1945) pp. 102-112.

60. Analytical theory of singular solutions of partial differential equations of the first order, Ann. of Math. vol. 46 (1945) pp. 120-143.

61. On the singular solutions of certain differential equations of the second order, Proc. Nat. Acad. Sci. U.S.A. vol. 32 (1946) pp. 255-258.

62. Abel's theorem and a generalization of one-parameter groups, Trans. Amer. Math. Soc. vol. 67 (1949) pp. 491-497.

63. Associative differential operations, Ann. of Math. vol. 51 (1950) pp. 756-765.

64. Differential groups and formal Lie theory for an infinite number of parameters, Ann. of Math. vol. 52 (1950) pp. 708-726.

65. Differential groups of order two, Ann. of Math., unpublished.

66. Subgroups of differential groups, Ann. of Math., unpublished.

67. Differential groups, Proceedings of the International Congress, Harvard, 1950, unpublished.

E. R. LORCH 